\title{
A Stable Charged Sphere in General Relativity.
}

\author{
N. K. SHARMA \\ Department of Physics, University of Rajasthan - Jaipur \\ (Nuovo Cimento, 48 A, 975 (1967))
}

Please read for eq. (14) p. 978,

$$
\mathscr{Y}^{a b} \Gamma_{a b \varrho}^{*}+\mathscr{Y}_{1 b}^{a b} g_{a \varrho}^{*}-\frac{1}{2} \mathscr{I}^{*}\left(F_{\mu \varrho}^{*} F^{* \mu 1}-\frac{1}{4} F_{\mu \nu}^{*} F^{* \mu v} g_{\varrho}^{* 1}\right)=0
$$

Please read for eq. (15) p. 979

$$
\mathscr{Y}^{a b} \Gamma_{a b c}+\mathscr{Y}_{1 b}^{a b} g_{a c}-\frac{1}{2} \mathscr{I}\left(F_{\mu c} F^{\mu 1}-\frac{1}{4} F_{\mu \nu} F^{\mu \nu} g_{c}^{1}\right)=0
$$

Please read for eq. (16) p. 979

$$
\mathscr{Y}^{a b} \Gamma_{a b}^{* 1}-\frac{1}{4} F_{a 1}^{*} F^{* a 1} \mathscr{I}^{*} g^{* 11}=0,
$$

Which is obtained as a consequence of multiplying eq. (14) by $g^{* 10}$ and with the use of boundary conditions

$$
F_{a b}^{*}=0
$$

The results and calculations arrived at in the two later notes (Nuovo Cimento, $52 \mathrm{~A}, 970(1967)$; $59 \mathrm{~B}, 121$ (1969)) remain unaltered except for minor numerical corrections. 